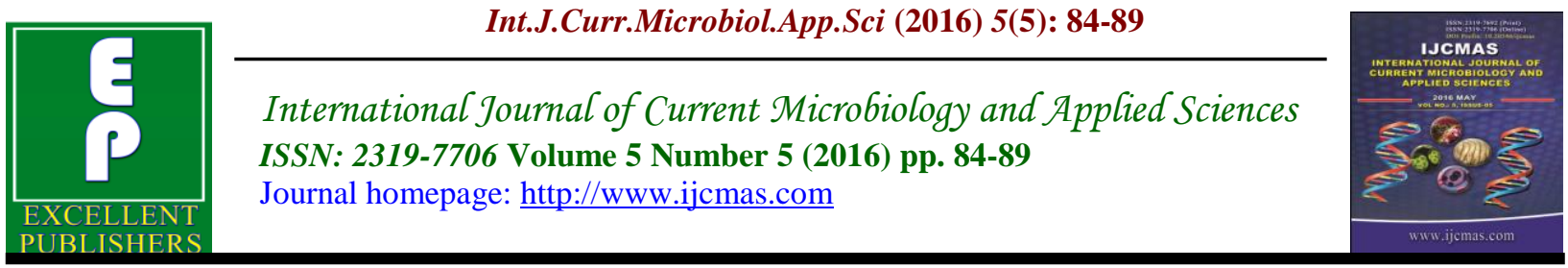

Original Research Article

http://dx.doi.org/10.20546/ijcmas.2016.505.009

\title{
A Tale of Two Novel Proteus Species-Proteus hauseri and Proteus penneri
}

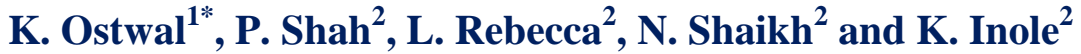 \\ ${ }^{1} \mathrm{GMC}$, Akola Maharashtra, India \\ ${ }^{2}$ Dr. Vaishampayan Memorial Government Medical College, Solapur, Maharashtra, India \\ *Corresponding author
}

\begin{tabular}{|c|c|}
\hline \multirow{7}{*}{$\begin{array}{l}\text { Ke y w o r d s } \\
\text { No swarming } \\
\text { motility, } \\
\text { Proteus } \\
\text { hauseri, } \\
\text { Proteus } \\
\text { Penneri. }\end{array}$} & A B S T R A C T \\
\hline & The present study reporting two different cases of two new Proteus species- \\
\hline & $\begin{array}{l}\text { reports a one month old male child with history of diarrhoea since six days. His } \\
\text { stool sample was send for microbiological examination and the second case is of }\end{array}$ \\
\hline & Ante natal care patient with urinary tract infection whose urine was send for \\
\hline & microbiological examination. Both of these isolates showed Non lactose fermenting \\
\hline & colonies. There was no swarming on Blood agar. Biochemical reactions revealed \\
\hline & $\begin{array}{l}\text { Proteus and both of these isolates were sent for further identification to National } \\
\text { Chemical Laboratory, Pune for further identification. First isolate was identified as }\end{array}$ \\
\hline Article Info & Proteus hauseri and second one as Proteus penneri. Both the patients were treated \\
\hline $\begin{array}{l}\text { Accepted: } \\
\text { 08 April } 2016 \\
\text { Available Online: } \\
10 \text { May } 2016\end{array}$ & $\begin{array}{l}\text { successfully with meropenem and were discharged few days later. As per our } \\
\text { knowledge, this is the third case of Proteus hauseri and few cases of Proteus } \\
\text { penneri have been reported so far. So, hereby we are presenting with two cases of } \\
\text { novel species of Proteus. Both of these species are very rare and as there was no } \\
\text { swarming on blood agar these isolates may be misdiagnosed. }\end{array}$ \\
\hline
\end{tabular}

\section{Introduction}

The genus Proteus along with genus Providencia and Morganella belongs to the tribe Proteae of the family Enterobacteriaceae. (Nita Pal et al., 2014) Hauser first noted the swarming nature of the organisms and divided the strains into the two species based on the speed of their ability to liquify gelatin: $P$. vulgaris liquefies gelatin "rapidly," and $P$. mirabilis does so "more slowly". (Hauser, 1892) Hauser also described "Proteus zenkeri," which neither swarmed nor liquefied gelatin, but he rescinded this particular finding 7 years later. The genus
Proteus currently consists of four named species (P.mirabilis, P.penneri, P.vulgaris, P.myxofaciens) and four unnamed genomospecies Genomospecies 3 was named Proteus hauseri to honor Gustav Hauser, the German microbiologist, who first described the genus. (Caroline Mohr O'Hara et al., 2000) In 1982, P. vulgaris biogroup 1 (genomospecies 1) was named Proteus penneri and was distinguished by its negative reactions for indole production, salicin fermentation and aesculin hydrolysis. The remaining two biogroups were both positive for indole pro- duction. However, 
biogroup 2 (genomospecies 2) was positive for salicin and aesculin and biogroup 3 was negative for salicin and aesculin. (Caroline Mohr O'Hara et al., 2000) Proteae are widespread in the environment and make up part of the normal flora of the human gastrointestinal tract. ${ }^{1}$ Proteus species are among the commonly implicated pathogens in hospital as well as community acquired infections. (Patrick Kwame Feglo et al., 2010) This pathogen has a diverse mode of transmission, and hence can cause infection in different anatomical sites of the body. Some of the incriminating sources of transmission are soil, contaminated water, food, equipments, intravenous solutions, the hands of patients and healthcare personnel Proteus ranks third as the cause of these infections, particularly in hospital-acquired cases. $\quad P$. mirabilis accounts for approximately $3 \%$ of nosocomial infections in the United States (Centers for Disease Control and Prevention, 1996) and is commonly isolated in clinical microbiology laboratories. Both $\mathrm{P}$. mirabilis and $\mathrm{P}$. vulgaris are widely distributed in the environment with reservoirs in soil, water, sewage and feces and have been isolated from the intestinal tract of mammals, birds and reptiles. Proteus penneri has been isolated from a number of diverse clinical sites, including abdominal wounds, urine, bladder calculi, epidural ulcers, bronchoalveolar lavage fluid, stool and infected conjunctiva. (Krajden et al., 1987) Indole negative Proteus species are invariably incorrectly identified as Proteus mirabilis, often missing out isolates of Proteus penneri. The urease enzyme of $P$. penneri is also believed to be a leading cause of kidney stone formation. (Pearson et al., 2008) Very few case reports of Proteus penneri have been reportd so far. And only one case report of Proteus hauseri by Caroline O' Hara has been reported. We are hereby reporting case reports of these
Proteus species.

\section{Materials and Methods}

Clinical profile of patient was recorded. Samples were subjected to microscopy, culture and antibiotic susceptibility testing according to Kirby Bauer disk diffusion method using disk of Hi- Media laboratories, Mumbai.

Case 1- A month old boy was brought by his parents with complaints of passing loose stools 8-10 times a day since 6 days. He had fever, persistant cry and was unable to accept feeds. There was no neck stiffness and other symptoms of meningitis. The baby was full term, born by caesarean section. His stool sample was send to microbiology department for Hanging drop, wet mount and culture.

Grossly, the stool was liquid, mucoid, greenish, non blood stained and was adherent to the container.

Microscopically,

Hanging drop- evidence of motile bacilli.

Wet mount reveaed abundant pus cells, few RBC's, cyst of Entamoeba histolytica and motile bacilli.

Culture was done on MacConkey's agar, TCBS and blood agar.

MacConkey's Agar showed, Non lactose fermenting, circular, smooth, irregular margin, 3-4mm colonies, catalase positive and oxidase negative colonies. Blood agar showed 3-4mm, circular, smooth, irregular margin, non haemolytic colonies without swarming.

TCBS showed greenish, 2-3mm, irregular margin colonies. 
Colonies from MacConkey's agar were subjected to biochemical reactions and Antibiotic susceptibility testing.

Case2- This case reports a 22 years female, with 6 months amenorrhoea came with complaints of fever, increase in frequency of micturation, pain and burning during micturation since 5 days. Her clean catched midstream urine sample was collected in morning and was send to microbiology department for microscopy and culture.

Grossly, urine was turbid, yellow, non odorous.

Microscopically on wet mount there were 56 pus cells/high power field and motile bacilli.

Urine was cultured on CLED agar. After overnight incubation, 3-4mm, yellow, circular, irregular margin colonies were seen that were catalase positive and oxidase negative. These colonies were subjected to biochemical reactions and Antibiotic susceptibility testing.

Repeat samples of both the patients were taken to rule out contamination.

The antibiotic susceptibility testing by Kirby Bauer disk diffusion method is as follows (Clinical and Laboratory standards Institute, 2014)

Case-1 Amikacin (9mm), ciprofloxacin (12mm), ceftriaxone (8mm), ceftazidime (11mm), ceftazidime plus clavulinic acid (14mm), meropenem (10mm), colistin (22mm).

Case-2 Amikacin (6mm), ciprofloxacin $(8 \mathrm{~mm})$, ceftriaxone $(6 \mathrm{~mm})$, ceftazidime $(12 \mathrm{~mm})$, ceftazidime plus clavulinic acid (14mm), meropenem (25mm).
Both the patients were treated with meropenem. The first patient was discharged after 10 days and second after seven days.

The biochemical reactions revealed Proteus species but the colonies on Blood agar were non swarming. So, both the strains were sent to National Chemical Laboratory, Pune for further identification.

The first isolate was confirmed as Proteus hauseri and second as Proteus penneri by 16sRNA PCR.

To prove the pathogenicity of these two isolates, intradermal inoculation was done in mice and after taking all necessary precautions. The site of intradermal inoculation was marked. And after 48 hours, a blister was present at injected site. It was around $1 \mathrm{~cm}$. Blister fluid was taken for culture and it also revealed same microorganisms.

\section{Discussion}

Case-1: In a study by Müller, $P$. mirabilis and $P$. penneri were isolated significantly more often from stools of patients with diarrheal disease than from healthy patients, leading him to speculate that these species may play a role in some diarrheal disease (Müller, 1986). Their true role, however, remains unsubstantiated. However, P.hauseri from stool has not been reported. Our isolate is from stool sample. Proteus generally shows swarming on blood agar. Only one article has been published in year 2000, by Caroline O Hara in which out of 52 isolates only two were $P$. hauseri. They have not mentioned whether swarming was present or not. Our isolate did not show swarming. Proteus hauseri can be distinguished from $P$. mirabilis, $P$. penneri and $P$. myxofaciens as it is positive for 
Indole production and all other three are negative. This is the main distinguishing feature by which $P$. hauseri can be distinguished. Anti- microbial susceptibility patterns, of O'Hara being susceptible to amikacin, ceftazidime, ciprofloxacin, imipenem, and tri- methoprim sulfamethoxazole. They were resistant to tetracycline. Our isolate was only sensitive to meropenem. The patient was treated successfully with meropenem and was discharged after ten days. Due to non swarming nature, many isolates of $P$.hauseri can be missed.

\section{Biochemical Tests of 2 Cases are as follows ${ }^{-9}$}

\begin{tabular}{|l|l|l|}
\hline Tests & Case1 & Case2 \\
\hline Indole & Positive & Negative \\
\hline Methyl Red & Positive & Positive \\
\hline Voges Proskauer & Negative & Negative \\
\hline Citrate(Simmons) & Was utilized & Was utilised \\
\hline Urease & Was hydrolysed & Was hydrolysed \\
\hline Triple sugar iron & Acid/acid with H2S & Acid/acid with H2S \\
\hline ONPG & Negative & Negative \\
\hline Nitrate & Was reduced to nitrite & Was reduced to nitrite \\
\hline Bile esculin & Not hydrolysed & Not hydrolysed \\
\hline Phenylalanine deaminase & Positive & Positive \\
\hline Ornithine decarboxylase & Negative & Negative \\
\hline Arginine dihydrolase & Negative & Negative \\
\hline Lysine decarboxylase & Negative & Negative \\
\hline DNAse & Negative & Negative \\
\hline
\end{tabular}

Figure.1 Showing Non Swarming Growth of Proteus hauseri on Blood Agar

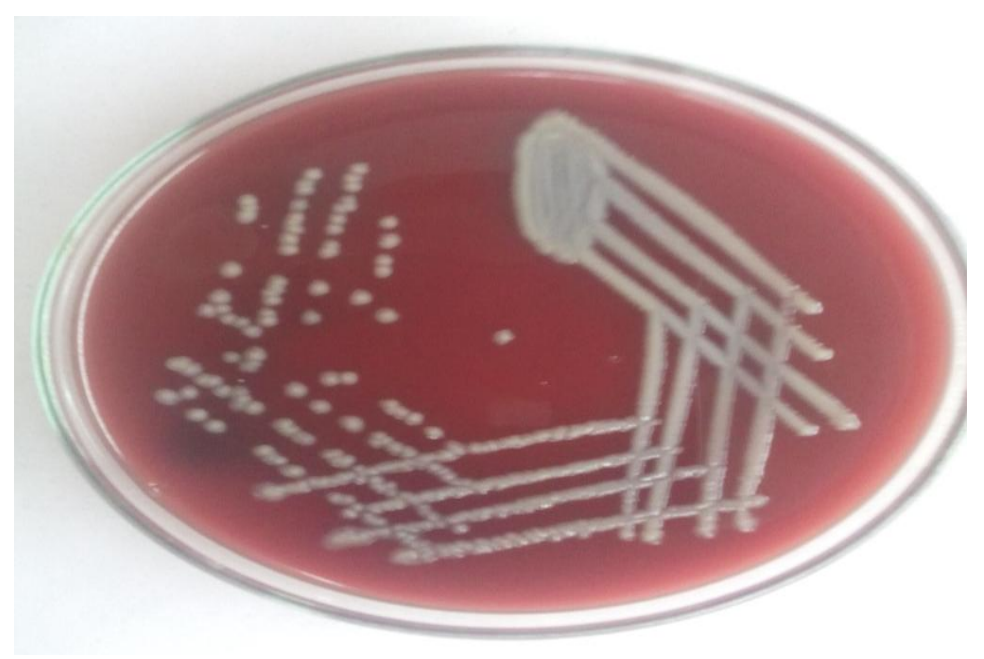


Figure.2 Showing Biochemical Reactions of Proteus hauseri

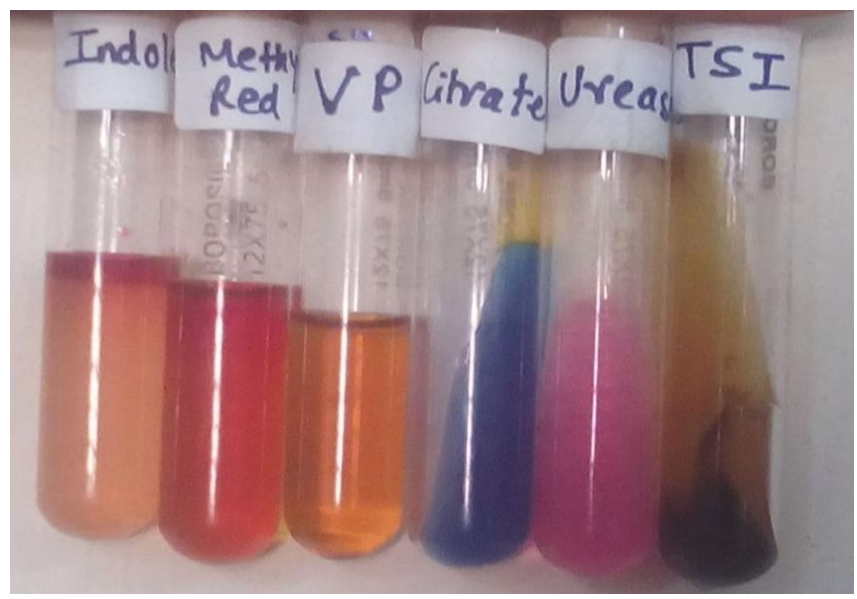

Case-2: $P$. penneri was absent in samples obtained from $<1,50-59$ and $70-79$ years age groups. (Jitendra Kumar Pandey et al., 2013) Very few case reports are there with $P$. penneri infection between 20-30 years of age. Our patient was 27 years old. $P$. mirabilis was the only Proteus species encountered in urine samples and this supported the finding that $P$. vulgaris and $P$. penneri infections of the urinary tract are rare. (Chung et al., 1999) P.penneri is mostly isolated from wound swab, and next from urine samples. Proteus spp. (P. mirabilis, $P$. vulgaris, and $P$. penneri) are important pathogens of the urinary tract and the primary infectious agents in patients with long-term indwelling urinary catheters. (Jacobsen et al., 2008) Our isolate is from urine sample from a non catheterized patient. P. penneri shows swarming on blood agar. However non swarming strains mainly on first isolation is seen in many cases (Janak Kishore, 2012). Multiple drug resistance in P.penneri is common. $P$. penneri was the most resistant among the recovered species. Our isolate was sensitive only to meropenem and resistant to all baseline antibiotics.

In conclusion, P.hauseri and P.penneri may not show swarming and hence could be missed. As they are most resistant Proteus species prompt diagnosis and early treatment is required to save the patient.

\section{References}

Caroline Mohr O'Hara, Frances, W., Brenner, Arnold, G., Steigerwalt, Bertha, C., Hill, Barry Holmes, Patrick, A.D., Grimont, Peter, M., Hawkey, John, L., Penner, J., Michael Miller, 1., Don, J., Brenner. 2000. Classification of Proteus vulgaris biogroup 3 with recognition of Proteus hauseri sp. nov., nom. rev. and unnamed Proteus genomospecies. Int. J. Systematic and Evol. Microbiol., 50: 1869-1875.

Caroline Mohr O'Hara, Frances, W., Brenner, Michael Miller, R. 2000. Classification, Identification, and Clinical Significance of Proteus, Providencia, and Morganella. Clin. Microbiol. Rev., 13(4): 534-546.

Centers for Disease Control and Prevention. 1996. National nosocomial infections surveillance (NNIS) report, data summary October 1986-April 1996, issued May 1996. Am. J. Infect. Control, 24: 381.

Chung, H.I., Hsueh, T., Chih, T. 1999. 
Prevalence of Proteus species in urinary tract infections in a regional hospital in Trinidad. 62: 438-442.

Clinical and Laboratory standards Institute. 2014. M100-S23. Performance standard for Antimicrobial susceptibility testing. Twenty four Informational supplement; Pennsylvania.

Hauser, G. 1892. Ueber das Vorkommen von Proteus vulgaris bei einer jauchigphlegmonösen eiterung nebst einigen bemerkungen zur biologie

Jitendra Kumar Pandey, Akanksha Narayan, Shikhar Tyagi. 2013. Prevalence of Proteus species in clinical samples, antibiotic sensitivity pattern and ESBL production. Int. J. Curr. Microbiol. App. Sci., 2(10): 253-261.

Krajden, S., Fuksa, M., Petrea, C., Crisp, L.J., Penner, J.L. 1987. Expanded clinical spectrum of infections caused byProteus penneri. J. Clin. Microbiol., 25: 578-579.

Müller, H.E. 1986. Occurrence and pathogenic role of MorganellaProteus-Providencia group bacteria in human feces. J. Clin. Microbiol., 23: 404-405.

Nita Pal, Nikita Sharma, Rajni Sharma, Saroj Hooja, Rakesh, K., Maheshwari. 2014. Prevalence of Multidrug (MDR) and Extensively Drug Resistant (XDR) Proteus species in a tertiary care hospital, India. ISSN: 2319-7706 Volume 3, Number 10, pp. 243-252.

Patrick Kwame Feglo, Stephen Yao Gbedema, Solomon Nii Armar Quay, Yaw Adu-Sarkodie, Clement Opoku- des Proteus. Münch

Wochenschr., 39: 103-105.

Jacobsen, S.M., Stickler, D.J., Mobley, H.L.T., Shirtliff, M.E. 2008. Complicated catheter-associated urinary tract infections due to Escherichia coli and Proteus mirabilis. Clin. Microbiol. Rev., 21(1): 26-59.

Janak Kishore. 2012. Isolation, identification \& characterization of Proteus penneri - a missed rare pathogen. Indian J. Med. Res., 135: pp 341-345.

Okrah. 2010. Int. J. Pharma Sci. Res., (IJPSR), Vol. 1(9): 347-352.

Pearson, M., Mohammed Sebaihia, Carol Churcher, Michael, A., Quail, Aswin, S., Seshasayee, Nicholas, M., Luscombe, Zahra Abdellah, Claire Arrosmith, Becky Atkin, Tracey Chillingworth, Heidi Hauser, Kay Jagels, Sharon Moule, Karen Mungall, Halina Norbertczak, Ester Rabbinowitsch, Danielle Walker, Sally Whithead, Nicholas, R., Thomson, Philip, N., Rather, Julian Parkhill, Harry, L.T., Mobley. 2008. Complete Genome Sequence of Uropathogenic Proteus mirabilis, a Master of both Adherence and Motility! Melanie. J. Bacteriol., p. 4027-4037.

Winn, W.C., Allen, S.D., Janda, W.M., Koneman, E.W., Precop, G.W., Schreckenberger, P.C. 2006. Koneman's color atlas and textbook of diagnostic microbiology. Chap. 7. 6th ed. New York: Lippincott; 2006. p. 392-429.

\section{How to cite this article:}

Ostwal, K., P. Shah, L. Rebecca, N. Shaikh and Inole, K. 2016. A Tale of Two Novel Proteus Species- Proteus hauseri and Proteus penneri. Int.J.Curr.Microbiol.App.Sci. 5(5): 83-89. doi: http://dx.doi.org/10.20546/ijcmas.2016.505.009 